\title{
Confocal Depolarized Dynamic Light Scattering: a Novel Technique for the Characterization of Nanoparticles in Complex Fluids
}

\author{
M.A.C. Potenza ${ }^{1,2}$, T. Sanvito ${ }^{3}$, F.P. Mariani ${ }^{1}$, P.G. Vekilov ${ }^{4}$, D. Maes ${ }^{5}$ \\ ${ }^{1}$ Department of Physics, \\ We present Confocal Depolarized Dynamic Light \\ University of Milan, via Celoria, \\ 16 - I-20133 Milan, Italy \\ ${ }^{2}$ CIMAINA, via Celoria, 16 - I- \\ Scattering as a novel optical method for the \\ 20133 Milan, Italy \\ characterization of nanoparticles in solution. The method \\ is validated in conditions of biological interest where \\ ${ }^{3}$ EOS Srl, Viale Ortles 22/4, I- \\ traditional optical methods fail. As a test case we study \\ 20139, Milan, Italy \\ ${ }^{4}$ Department of Chemical and \\ Biomolecular Engineering and \\ Department of Chemistry, \\ University of Houston, Houston, \\ Texas 77204, United States \\ ${ }^{5}$ Structural Biology Brussels, \\ SBB, Vrije Universiteit \\ Brussel, 1050 Brussels, Belgium \\ Corresponding author: \\ Marco A.C. Potenza \\ highly concentrated, strongly scattering, samples of \\ thermosensitive core-shell particles constituted by a \\ spherical PMMA core surrounded by a PNIPAM \\ network and, follow the kinetics of the processes \\ induced by temperature changes. We prove that through \\ the confocal optical scheme, the multiple scattering \\ contribution is reduced by orders of magnitude. \\ Moreover, the method allows the efficient \\ characterization of NPs with a superior accuracy in \\ particle size determination. Low degrees of \\ polydispersity can be easily characterized through an \\ adapted cumulant method.
}

marco.potenza@unimi.it

Keywords: Gold nanoparticles; Depolarized light scattering; core shell particles. 


\section{Introduction}

The use of nanoparticles (NPs) in medicine is considered a revolutionary improvement enhancing the diagnostic and therapeutic efficacy, with the possibility to provide a better control on the pharmacokinetics of drugs. One of the paramount features of NPs is the possibility to functionalize their surface in order to target with high precision a desired site (Mahon 2012). Synthetic polymer NPs have been given particular attention in view of their capability of responding to external stimuli such as temperature, $\mathrm{pH}$, etc., and modifying their structure in many different programmable ways (Mura 2013, Kumari 2010).

Despite their utmost importance in scientific and research environments, the actual clinical exploitation of synthesized NPs is limited. Manufacturing safe products, with adequate process control procedures, necessary from a clinical point of view, is still a challenge (Park 2013, Desai 2012, Nichols 2012). On the one hand, the possibility to use a broad variety of NPs with different dimensions, size distribution, shape, surface chemistry, density, drug loading and stability offers multiple advantages but on the other hand adequate characterization procedures in vitro and in vivo (Shang 2014, Albanese 2012, Treuel 2014, Moore 2015) are required that are beyond the capabilities of the standard characterization methods.
Although current methods can be applied to measure the response of NPs to external stimuli under ideal conditions in laboratory set-ups, they cannot be used in less ideal environments such as in complex biological fluids, where it would be important to grasp the NPs specific evolution and modifications (Xie 2015, Itoh 2015, Mukherjee 2008, Huang 2013). In this paper, we will present a novel technique for the characterization of NPs in solution.

Nowadays, the most common method to characterize NPs in solution is dynamic light scattering (DLS). Although DLS is very reliable and can be used by non-specialized personnel, it does not fill the gap discussed above and cannot be used to fully characterize NPs in complex fluids. The presence of a heterogeneous, polydisperse submicron component in the fluid introduces a serious limitation on the use of DLS. Extracting signals solely due to the test particles is impossible. Moreover, the possibility for the light to undergo multiple scattering events mixes up the signals even more. Recently, some groups have envisaged the possibility to rely on the internal optical anisotropy of NPs by measuring the depolarized light only (Balog 2014, Balog 2015). By exploiting Depolarized DLS (DDLS) schemes, the contribution of the background component of the complex fluid is appreciably depressed, and the presence of a few, small engineered NPs can be 
evidenced and characterized. Nonetheless, the use of DDLS has been strongly limited because of the multiple scattering issue, which typically hide the pure depolarized signal generated by the anisotropic particles.

Here we present an adapted version of the DDLS technique that is able to overcome the limitations typical of both DLS and DDLS and which allows the efficient characterization of NPs with a superior accuracy in particle size determination (Potenza 2010, Potenza 2011). Our approach is based upon the analysis of the forward, depolarized scattered fields generated from a collection of NPs illuminated by a tightly focused laser beam. Collecting the light emerging from the sample in the forward scattering direction through a strict confocal optical scheme, the multiple scattering contributions are reduced by orders of magnitude. Moreover, by selecting only the pure depolarized component, turbid samples can be studied for the first time.

In this work, we describe the experimental method. We prove the feasibility of measurements in known samples affected by strong multiple scattering, and we study the effect of polydispersity. We also present results of measurements performed on suspensions of core-shell particles constituted by a spherical PMMA core surrounded by a PNIPAM network that is thermosensitive. Future applications of the method are discussed.

\section{Materials and Methods}

2.1 The Confocal Depolarized Dynamic

Light Scattering (CDDLS)

The CDDLS method was introduced as an extension of the traditional DDLS method (Degiorgio 1994, Degiorgio 1997), with the aim to overcome the typical limitations arising from multiple scattering. The ultimate reason why the traditional DDLS fails is the fact that optical anisotropy gives rise to a depolarized scattered amplitude that is orders of magnitude smaller than the polarized scattered one. Therefore, the probability to detect polarized light which suffered two (or more) scattering events is comparable or even larger than the probability to detect depolarized scattered light. As a result, DDLS can only be applied in highly specific conditions, which are not of interest for most applications.

The fundamentals of the CDDLS method and the experimental apparatus are described in details in (Potenza 2010, Potenza 2011). Here we just recall the basics of the method, and briefly describe the optical layout. Depolarized light scattering relies on the measurement of the light scattered by optically anisotropic particles. The optical anisotropy can be a consequence of the internal crystalline structure of the particles or the strong shape anisotropy, as for highly elongated particles (Van de Hulst 1957, Pecora 1976, Bohren 1983). As in traditional 
DLS, DDLS takes advantage of the continuous Brownian motions of the particles immersed in a fluid: whereas the translational Brownian motions can be measured with the standard DLS method, the rotational Brownian motion randomly changes the orientation of the particles resulting in fluctuations in the depolarized scattered fields. For a collection of monodisperse anisotropic particles, these intensity fluctuations have a characteristic decorrelation time $\tau$ that is equal to the inverse of six times the rotational diffusion coefficient $\Theta$. Using the Stokes-Einstein relation:

$\Theta=\frac{k_{B} T}{8 \pi \eta R^{3}}$

where $k_{B}$ is the Boltzmann's constant, $T$ the absolute temperature and $\eta$ the kinematic viscosity of the fluid surrounding the particle an estimate of the radius $R$ of the particle can be derived based on the experimentally determined characteristic decorrelation time $\tau:$

$R^{3}=\frac{3 k_{B} T}{4 \pi \eta} \tau$

Because the rotational time constant depends on the third power of the radius small differences in radius will have a large impact on the time constants. Hence, rotational diffusion offers a superior resolution in particle sizing as compared to translational diffusion. This is of particular importance in applications where the NPs under study are monodisperse, and minute changes of radii have to be studied. We will demonstrate below that CDDLS is sensible to changes in radii of less than $1 \mathrm{~nm}$ for particles $100 \mathrm{~nm}$ in diameter. Moreover, the presence of a small poly-dispersity in the size distribution is amplified by this third power dependence. Note that in this case the traditional cumulant method is no longer valid, and a dedicated analysis is necessary (Potenza 2012).

In Fig. 1 we schematically represent the optical layout. A laser beam (He-Ne JDS Uniphase 1100; $632.8 \mathrm{~nm}$ wavelength, 20 $\mathrm{mW}$ power) is linearly polarized through a Glan-Thompson prism, and tightly focused through a long distance microscope objective (Nachet 20x, NA $=0.30$ ) into a plane parallel quartz cell $2 \mathrm{~mm}$ thick filled with the sample. The focal spot is approximately $5 \mu \mathrm{m}$ in diameter, with a transverse intensity distribution which is approximately Gaussian. The emerging light is collected and collimated through an identical microscope objective followed by an analyzer (GlanThompson prism) almost at extinction conditions. In such a way, most of the depolarized scattered light passes, while the transmitted beam is attenuated approximately 1000 times. Finally, the collimated beam is focused into a monomode optical fiber (FS$\mathrm{SN}-3224$, core diameter $4.3 \mu \mathrm{m}$ ), in order to select the light emerging from the focal region (see Potenza 2010 for details). Light is then sent to a photomultiplier tube (PMT; model PMT120-OP), which samples the 
intensity as a function of time. The for single particle measurements as in (Maes superposition of the depolarized fields 2015), the deterministic approach (option 2) scattered by the particles within the focal is more advantageous. In our set-up, a multiregion and the attenuated transmitted beam tau correlator

(FLEX-02-01-D, gives rise to interference effects which Correlator.com, CA) with a minimum lag fluctuate in time with the time constant of the rotational Brownian motions of the NPs in the fluid. There are two ways to analyze the obtained intensity signal: 1) via the calculation of the intensity correlation function, exactly as it is done in the DLS method; 2) via the direct analysis of the raw intensity function. In case one deals with a number of scatterers within the small time of $3.3 \mathrm{~ns}$ provides as well the normalized correlation function evaluated in 1120 lag times as the history of the intensity sampled each 0.17 s. For accessing correlation times up to about $1 \mathrm{~s}$, the entire optical line was put into a tube, limiting the unavoidable air turbulence. The latter causes slight deflections dramatically affecting the intensity of the light collected by the optical scattering volume a statistical approach fiber.

(option 1) is more appropriate. In contrast,

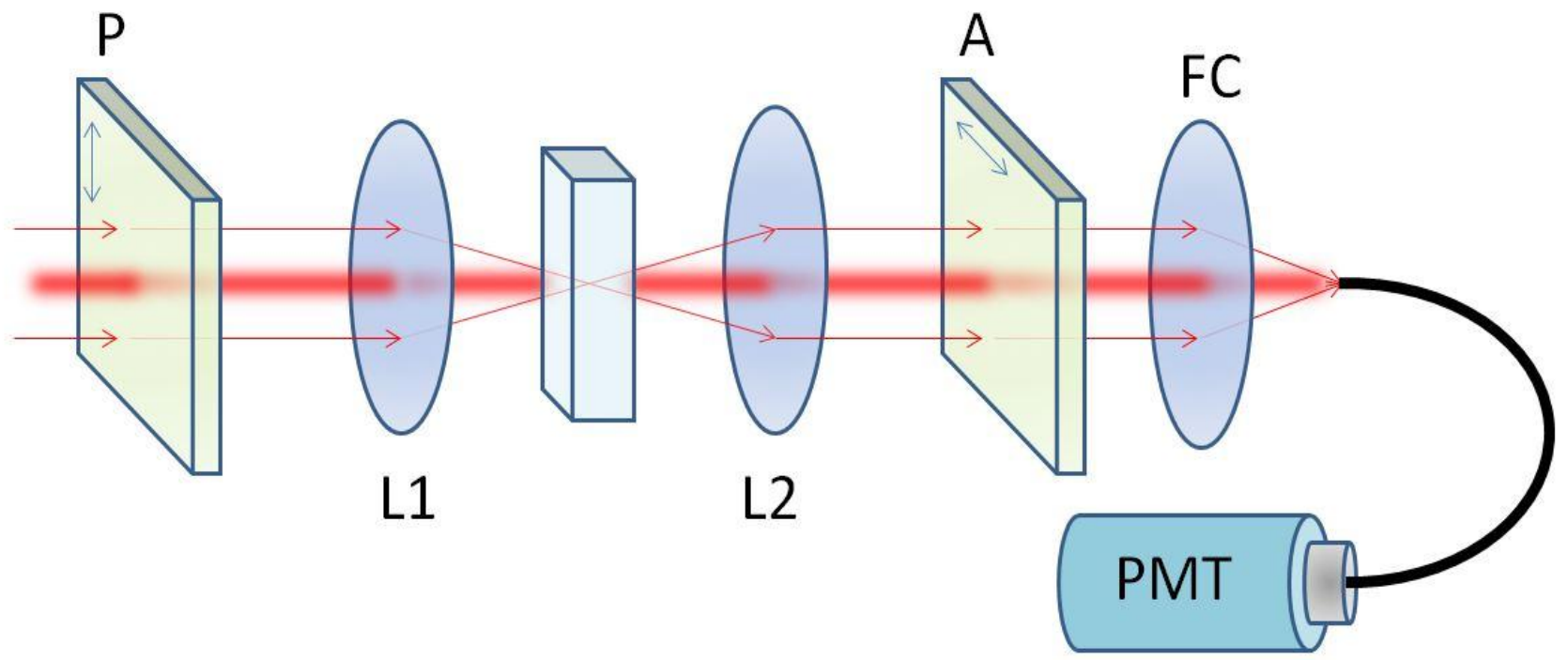

Figure 1: Schematic of the experimental device. The laser beam is polarized by polarizer P, and focused into the sample cell by the microscope objective L1. The emerging light is collected through a symmetric objective L2, and the collimated beam passes through the analyzer A to the lens FC. The light is then launched into the monomode fiber connected to the PMT. 
We stress that the confocal condition guarantees that only light emerging from the focal spot is properly sent through the optical fiber, damping strongly any other contribution. As such multiple scattered light, which is essentially generated in the whole cell after the first scattering event occurred in the focal spot (where the intensity of light is much higher than outside the focal region), is dramatically reduced.

Finally, in order to independently check the turbidity of the samples at high concentration, a simple measurement of the turbidity of the cell has been done prior to each CDDLS measurement. The cell was illuminated by a collimated laser beam and the intensity measured with a photovoltaic cell in the far field. Monitoring the turbidity allows to have an independent characterization of the concentration of the samples during the CDDLS measurements.

\subsection{The samples}

We performed two kinds of measurements: 1) measurements representing a proof of principle of the method, performed with calibrated, monodisperse suspensions of spheres endowed with optical anisotropy; 2) measurements following the evolution of a suspension of thermosensitive core-shell particles, of which the size shrinks with increasing temperature.
For the first type of measurements suspensions of monodisperse, calibrated, spherical NPs were prepared at different concentrations, volume fraction ranging from $10^{-4}$ to $10^{-2}$, in pure, filtered water. The NPs are composed of MFA, which do have an internal crystalline structure (Degiorgio 1994, Degiorgio 1997). They have been accurately characterized with traditional DLS and a hydrodynamic diameter of $95 \mathrm{~nm}$ was obtained. Polydispersity wasn't detectable with DLS. For the second type of experiments quasimonodisperse suspensions of spherical core-shell particles were prepared. The samples were quasi-monodisperse suspensions of spherical core-shell particles. The particles consist of a spherical PMMA core $50 \mathrm{~nm}$ in diameter surrounded by a PNIPAM network encapsulating a few, very small palladium nanoparticles. The latter is responsible for the high optical anisotropy of the system. Thanks to the thermal properties of PNIPAM, the size of the particle can be reduced by increasing the temperature of the solution up to $32^{\circ} \mathrm{C}$, where the size stabilizes. The system is fully reversible, also after the temperature has been increased above $32^{\circ} \mathrm{C}$. The tested particles had approximate radii $80 \mathrm{~nm}$ at $20^{\circ} \mathrm{C}$ and a polydispersity of the order of $10 \%$. These samples were produced by the group of Prof. M. Ballauff under cryo-TEM control 
as discussed in (Dingenouts 1998, Crassous 2006, Crassous 2008). Prior to CDDLS data collection, the particles samples were characterized by classical DLS at $20^{\circ} \mathrm{C}$ and the results obtained are displayed in Fig. 2.

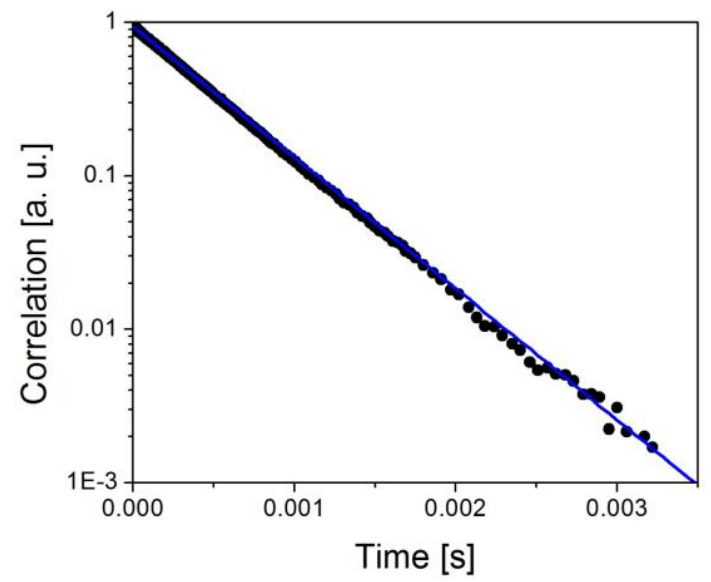

Figure 2: Traditional $90^{\circ}$ DLS results obtained on the core-shell particles. The continuous line represents a pure exponential decay.

\section{Results}

\subsection{Measurements on MFA}

Measurements performed on fully characterized monodisperse MFA spheres served as a proof of principle of the method, in particular its capability of dealing with multiple scattered light. An example of a correlation function obtained with this sample at a volume fraction of 0.098 is shown in Fig. 3.a. A fast decay mode is evident, likely caused by the rotational Brownian motions (see Potenza 2010). A slower, non-exponential decay mode is present due to spurious fluctuations of the transmitted intensity. With a stronger focusing the slowest mode would be exponential delivering the important information about the translational diffusion as already reported in (Potenza 2010). This is not possible here, because of the relatively large focal spot causing the laser fluctuations to decorrelate the signals on times shorter than the characteristic times for Brownian translational motions. The condition used in this work is suitable for the measurements of the fast modes we are interested in. In order to extract the genuine depolarized signal, a fit of the slow mode can be easily obtained, at least below $10^{-2} \mathrm{~s}$ which is the contribution affecting the fast mode. An exponential decay is adopted here. 

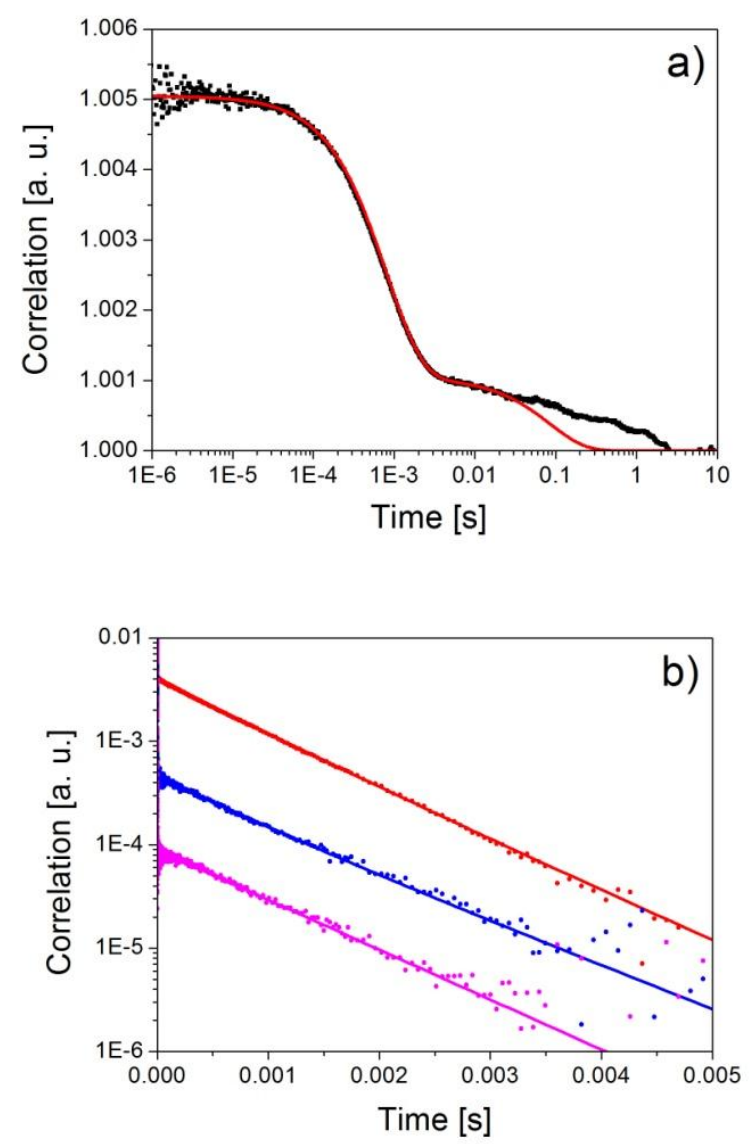

Figure 3: a) An example of a typical correlation curve obtained with monomeric MFA spheres $95 \mathrm{~nm}$ in diameter. The continuous line represents a double exponential decay. It demonstrates the almost exponential decay of the fast mode and the non-exponential decay of the slow one. b) Examples of the fast modes obtained with three volume fractions. From top to bottom, 0.098 (based on the data shown in Fig. 3.a.), 0.020, 0.004. A log-lin scale is adopted here. Continuous lines indicate the best-fitted curves including polydispersity.

The rotational contribution can then be extracted and is shown on a log-lin scale in Fig. 3.b. for three different volume fractions: 0.098 (same data set as in Fig. 3.a, up to 5 $\left.10^{-3} \mathrm{~s}\right), 0.020,0.004$. As expected the amplitudes of the correlations functions are proportional to the concentrations, as expected (Potenza 2010). The size of the particles is reproduced with an accuracy of 2 $\mathrm{nm}$. A deviation from a pure exponential decay is evident. This can be attributed to the small polydispersity of the sample, which was not detectable with DLS. This proves the high sensibility to polydispersity of the method a consequence of the $\mathrm{R}^{3}$ dependence of the decorrelation time $\tau$ (equation 2). Analysis of the data taken into account the polydispersity is a non-trivial issue. The common cumulant method adopted for traditional DLS must be 
reconsidered due to the $\mathrm{R}^{3}$ dependence mentioned above. By following the method described in details in (Potenza 2011), a polydispersity of approximately $5 \%$ is obtained from our data.

For testing the reliability of the system in getting rid of the multiply scattered light we have repeatedly changed the sample concentration and compared the results. We spanned concentrations ranges, and, therefore, turbidity ranges, by more than a factor 100. Always, results within less than $5 \%$ error were obtained. In Fig. 4 we report the amplitude of the fast mode of the correlation function versus the concentration. As expected (Potenza 2010) this amplitude scales as the number concentration and this over three orders of magnitude. Actual values for the concentration depend on the size and relative refractive index of the particles: for example, core shell particles $80 \mathrm{~nm}$ in diameter can be measured for volume fractions from $10^{-4}$ up to $10^{-2}$. For higher concentrations, multiple scattering begins to affect the data, as evidenced by the shorter correlations times. Note that traditional DLS and DDLS cannot deal with these high concentrations due to the multiple scattering contributions.

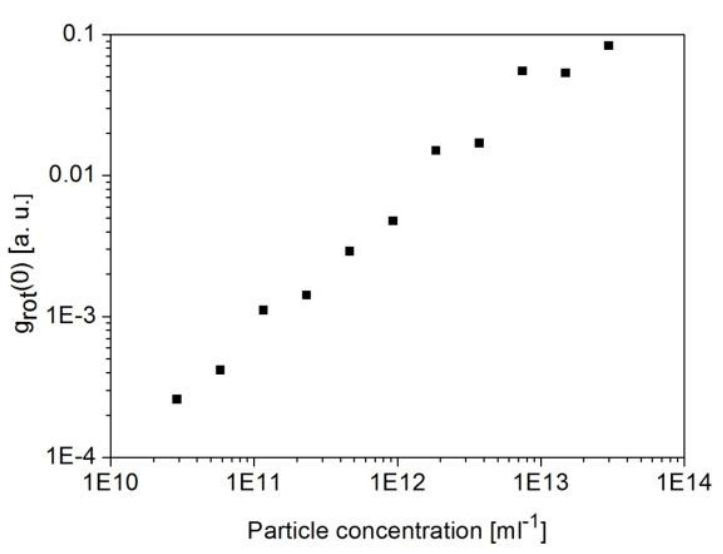

Figure 4: The amplitude of the correlation function measured at zero lag time, plotted against the concentration of the MFA sample, expressed as the number concentration.

Notice that the results shown in Fig. 4 can be interpreted as a calibration curve. Recalling the fundamentals of depolarized light scattering and the basics of the technique (Berne 1976, Potenza 2012), the amplitude of the correlation function is related to the number density, the particle polarizability and the anisotropy factor. Therefore, the calibration shown in Fig. 4 can be exploited to determine one of these parameters once the others are known. This scaling is valid until the rejection of the multiply scattered light is effective. 


\subsection{Thermosensitive samples}

The analysis of thermosensitive suspensions is one of the typical applications of the CDDLS method; one follows the kinetics of a reaction in a colloidal suspension undergoing physico-chemical effects. Here we report a study on core shell particles. In this case, temperature is the external trigger for the reaction that alters the properties of the particles.

In Figure 5 we show a typical correlation function of the core shell particles at $20^{\circ} \mathrm{C}$ represented in a log-lin plot after subtracting the slow mode (see above). The discrepancy from an exponential decay (represented by the dashed line) clearly reflects the polydispersity of the sample. This polydispersity wasn't detectable with DLS (Fig 2) where the decay appeared to be purely exponential. Hence, our setup enables to follow the kinetics of processes induced by temperature increase by measuring both the hydrodynamic radii, and the polydispersity of the sample. In addition to the CDDLS measurements, accurate DLS measurements with calibrated spheres have been done in order to monitor the water viscosity change with temperature. As such the size of the particles at different temperatures can be accurately determined. In Figure 6.a we report the hydrodynamic radii of the core shell particles during a temperature scan. As expected we observe a transition at $\mathrm{T}=32^{\circ} \mathrm{C}$ (Dingenouts 1998, Crassous 2006, Crassous 2008). The polydispersity during the temperature scan has been evaluated following the approach described above, and the results are reported in Fig. 6.b. Note that again the transition is clearly visible at $\mathrm{T}=32^{\circ} \mathrm{C}$. As already mentioned above traditional DLS is not sensible enough to characterize polydispersity as the CDDLS proves to be.

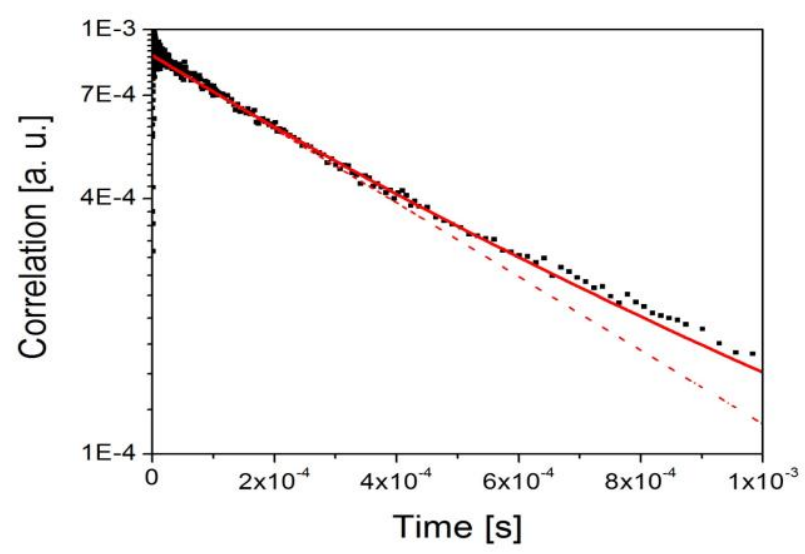

Figure 5: Typical correlation curve obtained with the core-shell particles. The nonexponential decay is evidenced by comparing data to the exponential curve (dashed line). The continuous line is the best-fitted curve including polydipsersity. 
We performed several temperature scans on the samples at different concentrations. No significant differences have been observed upon changing the concentration, neither during different repetitions of the coolingwarming cycles. Moreover, the transition is completely reversible.
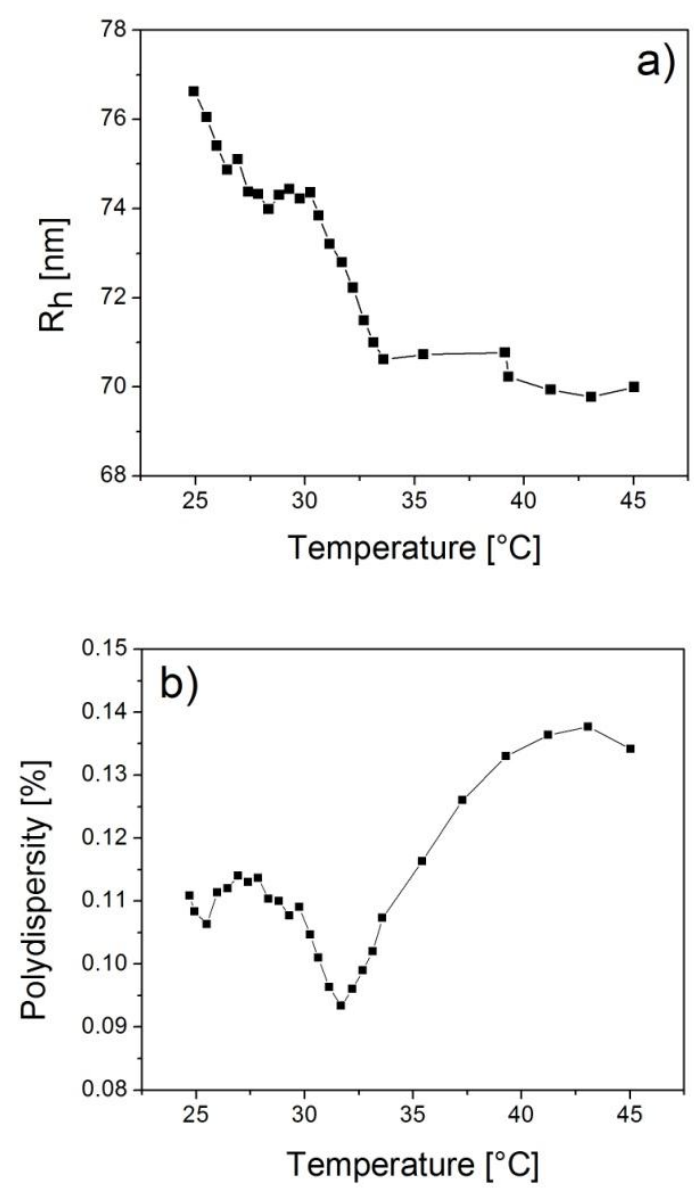

Figure 6: a) Rotational hydrodynamic radii of the thermosensitive core shell particles as a function of the temperature. b) The polydispersity of the core shell particles as a function of the temperature.

\section{Conclusions}

In this work we provide a proof of principle that the novel technique CDDLS is capable to overcome some of the major limitations of traditional DLS and DDLS. The CDDLS method can be applied to study a suspension of colloidal, optically anisotropic particles often found in biological liquids. The contributions to the scattered light from all amorphous components are suppressed. Moreover, thanks to a strong reduction of the multiple scattered components, it can operate with any kind of sample, in contrast to the classical DDLS method that requires 
samples in very specific conditions. In addition our results on thermosensitive coreshell particles demonstrate that the CDDLS method is particularly suited to study particle size changes triggered by external conditions, and to follow size and polydispersity changes. Currently, we are optimizing the CDDLS method for applications in a microgravity environment. We will use the technique to study the early stages of nucleation of protein crystals. A preliminary study is described in (Maes 2015). The method is capable of identifying nanocrystals in an environment constituted by amorphous protein clusters. Furthermore, work is in progress to apply CDDLS for the characterization of metallic NPs endowed with shape anisotropy, immersed in a complex fluid. 


\section{References}

Albanese A., Tang P. S., Chan W. C. The Effect of Nanoparticle Size, Shape, and Surface Chemistry on Biological Systems. Ann. Rev. Biomed. Eng. 14, 1-16 (2012)

Balog S. et al., J. Phys. Chem. C 118, 1796917974 (2014)

Balog S. et al., Nanoscale 7, 5991-5997 (2015)

Pecora R, Berne B.J., Dynamic Light Scattering, Dover Publications, Inc., 1976

Bohren C.F., Huffmann D.R., Absorption and Scattering by Small Particles, Wiley., Inc. 1983

Crassous J.J., et al. J. Chem. Phys., 125, 204906 (2006)

Crassous J.J., et al. Colloid Polym. Sci., 286, 805 (2008)

Degiorgio V., Piazza R., Bellini T., Visca M., Adv. Colloid Interface Sci. 48, 61 (1994)

Degiorgio V., Bellini T., Piazza R., Mantegazza F., Physica A 235, 279 (1997)

Desai N. Challenges in Development of Nanoparticle-Based Therapeutics. AAPS J. 14, 282-295 (2012)

Dingenouts N., Norhausen C., Ballauff M., Macromolecules 31, 8912 (1998)

Huang J., Zong C., Shen H., Cao Y., Ren B., Zhang Z. Tracking the Intracellular Drug Release from Graphene Oxide using SurfaceEnhanced Raman Spectroscopy. Nanoscale 5, 10591-10598 (2013)

Itoh N., Santa T., Kato M. Rapid evaluation of the quantity of drugs encapsulated within nanoparticles by high-performance liquid chromatography in a monolithic silica

column. Anal. Bioanal. Chem. 407 6429- Thermodynamics of Multistep Nucleation $6434(2015)$

Copyright 2015 KEI Journals. All Rights Reserved

Volume 2, issue 2
Kumari A., Yadav S., Yadav S. C. Biodegradable polymeric nanoparticles based drug delivery systems. Colloids Surfaces B: Biointerfaces 75, 1-18 (2010)

Maes D., Vorontsova M.A., Potenza M.A.C., Sanvito T., Sleutel M., Giglio M., Vekilov P.G.. Do proteins nucleate within dense liquid clusters? Acta Cryst F 71, 815-822 (2015)

Mahon E., Salvati A., Baldelli Bombelli F., Lynch I., Dawson K. A. Designing the nanoparticle-biomolecule interface for "targeting and therapeutic delivery". $J$. Contr. Rel. 161,164-174 (2012)

Moore T. L., et al., Nanoparticle colloidal stability in cell culture media and impact on cellular interactions, Chem. Soc. Rev. 2015, DOI: $10.1039 / \mathrm{c} 4 \mathrm{cs} 00487 \mathrm{f}$

Mukherjee B., Santra K., Pattnaik G., Ghosh S. Preparation, characterization and in-vitro evaluation of sustained release protein-loaded nanoparticles based on biodegradable polymers, Int. J. Nanomed. 3, 487-496 (2008)

Mura S., Nicolas J., Couvreur P. Stimuliresponsive nanocarriers for drug delivery. Nat. Mater. 12, 991-1003 (2013)

Nichols J. W., \& Han Bae Y. Odyssey of a cancer nanoparticle: from injection site to site of action. Nano Today 7, 606-618 (2012)

Park, K. Facing the truth about nanotechnology in drug delivery. ACS Nano 7, 7442-7447 (2103)

Pecora R., Berne B.J. Dynamic light scattering, Dover Publications, Inc., 1976 Potenza M.A.C., Sanvito T., Alaimo M.D., Degiorgio V., Giglio M., Eur. Phys. J. E, 31, 69 (2010)

Potenza M.A.C. et al., Kinetics and 
and Self-Assembly in Nanoscale Materials, pp. 61.78: John Wiley \& Sons, Inc (2012)
Van de Hulst H.C., Light Scattering By Small Particles, Dover Publications, Inc. 1957

Treuel L., Eslahian K. A., Docter D., Lang T., Zellner R., Nienhaus K., Nienhaus G. U., Stauber R. H., Maskos M. Physicochemical characterization of nanoparticles and their behavior in the biological environment. Phys. Chem. Chem. Phys. 16, 15053-15067 (2014)
Xie L., Beyer S., Vogel V., Wacker M.G., Mäntel W. Assessing the drug release from nanoparticles: Overcoming the shortcomings of dialysis by using novel optical techniques and a mathematical model, Int. J. Pharm., 488, 108-119 (2015) 